\title{
Learning to be selfish? A large-scale longitudinal analysis of Dictator games played on Amazon Mechanical Turk
}

\author{
By ANTONIO A. ARECHAR AND DAVID G. RAND*
}

We investigate the role of experience playing the Dictator Game (DG) on allocations by aggregating data from 37 studies conducted on Amazon Mechanical Turk over a 7-year period. While prior evidence has shown a correlation between experience on Amazon Mechanical Turk and selfishness, it is unclear to what extent this is the result of selection versus learning. Examining a total of 27,266 decisions made by 17,791 unique individuals, our data shows evidence of significant negative effects of both selection and learning. First, people who participated in a greater total number of our studies were more selfish, even in their first game - indicating that people who are more likely to participate in our studies are more selfish. Second, a given individual tends to transfer less money over successive studies - indicating that experience with the DG leads to greater selfishness. These results provide clear evidence of learning even in this non-strategic social setting.

Final version to be published in:

Journal of Economic Psychology

Volume 90, June 2022, 102490

doi: https://doi.org/10.1016/j.joep.2022.102490

Keywords: dictator game; selection; experience; learning; cooperation; decision-making; mturk JEL Classification: C72, C80, C90, D00.

Declarations of interest: none.

\footnotetext{
We thank the helpful comments by the Editor and two anonymous referees (Journal of Economic Psychology). Anonymized data and code are available on https://osf.io/juc7e/

* Arechar: Center for Research and Teaching in Economics; Centre for Decision Research and Experimental Economics, University of Nottingham; Sloan School of Management, Massachusetts Institute of Technology (e-mail: antonio@arechar.com); Rand: Sloan School of Management and Department of Brain and Cognitive Sciences, Massachusetts Institute of Technology (e-mail: drand@ mit.edu).
} 


\section{Introduction}

Although classical economic models assume that people are purely self-interested, this assumption is contested by a great deal of empirical evidence. Countless experiments have shown that people will incur costs to benefit others, even in one-shot anonymous settings where there are no strategic benefits of doing so. This observation is demonstrated most simply using results from the Dictator Game (DG), in which subjects regularly share some of their endowment with an anonymous stranger (Camerer \& Thaler, 1995; Guala \& Mittone, 2010).

Furthermore, while a great deal of work has examined learning in strategic settings (Fudenberg \& Levine, 1998; Benndorf et al., 2017, Conte et al., 2019), learning in non-strategic settings has received less attention. This is perhaps not surprising, as on first examination it may seem as though there is nothing to learn. In the DG, for example, if giving is driven by purely outcome-dependent preferences (e.g. inequity aversion or altruism), then there is no scope for learning whatsoever as the other player takes no action. Thus, one might expect an individual's giving not to vary systematically across multiple DG trials.

Yet, empirical evidence has found a negative effect of experience on subsequent prosociality. In Achtziger et al. (2015), participants played 12 consecutive rounds of the DG and the overall amount they shared decreased steadily over time (also suggesting that pro-social behavior may be short-lived as sessions lasted about 50 minutes). In a meta-analysis, Engel (2011) also finds that multiple trials have a clear negative effect on giving.

This negative trend has not only been documented within the boundaries of a single session. When comparing the allocations given in an experimental session with a subsequent one, separated by as little as 4 hours or by as much as 7 months, there is evidence of a decline in giving (Brañas-Garza et al., 2013; Brosig-Koch et al., 2017; McAuliffe et al., 2018; Sass et al., 2018). Relatedly, based on the number of previous participations in (allocation) lab experiments, Matthey \& Regner (2013) find that the amount subjects allocate to themselves tends to increase as they gain more experience. A similar relationship in the realm of online experiments on Amazon Mechanical Turk (MTurk) is also reported by Arechar et al. (2017) and Capraro et al. (2014).

Despite this evidence, it is unclear if the negative relationship between experience and DG giving generalizes to more representative populations, more trials, and longer time horizons. Most of the studies described above rely on small and somewhat-homogeneous samples of college students, the results are limited to a few repetitions or are collected within a short period of time and, crucially, for the studies where 
experience is only measured through self-reports, the correlation could be driven by selfish people participating in more studies, rather than actual changes with experience.

To shed further light on these issues, we aggregate the results of all online DG studies conducted by our lab on Amazon Mechanical Turk (MTurk) between 2012 and 2018 and look at the temporal dynamics of the resulting 27,266 allocations made by 17,791 individuals. ${ }^{1}$ Because we can track participants' number of trials over time and their total number of DGs played, this dataset allows us to explore, distinguish, and provide novel insights into the roles of selection versus learning on DG giving (although the nature of such distinctions is not experimental or randomized, only longitudinal and correlational).

We find clear evidence of both selection and experience effects: while on average selfish people tend to be more likely to participate in our DG studies, we also see that average giving decreases across successive studies for a given individual. Furthermore, we find that this experience effect is not explained by changes in participants' beliefs about whether their recipient is real and compensated.

The remainder of this paper is structured as follows. The second section details the methods followed to curate the data. In Section 3 we report the results of our research. Finally, in Section 4 we present a discussion of the results and make concluding remarks.

\section{Methods}

First, we aggregated data from all 39 DG studies conducted on MTurk by our lab between 2012 and 2018, but excluded one where the recipient was associated with their political identity and another one with a "take" framing. In all of the remaining 37 studies (27,266 decisions made by 17,791 unique individuals; see Table S1 of the Online Appendix for details of each study), participants made an incentivized single one-shot unilateral decision about how to divide a sum of money between themselves and another anonymous person. We then use participants' unique MTurk ID number to connect their behavior across multiple studies.

Studies varied in the endowment allotted to the participants (from $\$ 0.10$ to $\$ 1.40$ ). To account for the differences in endowment and streamline comparisons, our dependent variable is the fraction shared in the

\footnotetext{
${ }^{1}$ Overall, participants share $30.47 \%$ of their endowment with an anonymous other, which is quite close to the $28.35 \%$ reported meta-analytically by Engel (2011) using mostly conventional samples. Yet, subjects in our sample tend to be older $($ mean $=34.37$ years old; s.d. $=11.25$; $\min .=18 ; \max =87)$, can complete over a dozen trials, and have a timehorizon between trials of as little as 4 minutes and as long as 5 years and 11 months. Data available on https://osf.io/juc7e/.
} 
DG (i.e. absolute amount given divided by endowment size). To further account for this and other studyspecific variations (e.g. other treatments or games that occurred prior to the DG), we include study-specific dummies in all the analyses (although results are qualitatively equivalent if study-specific dummies are omitted. See Tables S2-S4 of the Online Appendix for detailed results of all statistical analyses).

Unless otherwise stated, we use linear regressions with two independent variables to dissociate selection from experience: the total number of DGs played by the participants (a slope capturing selection), and the number of DGs played prior to the current decision (a slope capturing experience). Robust standard errors are clustered on participants' ID to account for the non-independence of repeated observations from the same MTurk worker. Note that by selection, we do not just mean the choice of whether to select into a given experiment when presented with the opportunity. Rather, we mean selection in a more general sense that captures all factors that lead someone to participate or not in a given study, such as how often they use MTurk in general, their preference for this kind of tasks on MTurk, their attitude toward our requester account, etc. The key point of this part of the analysis is to separate out all of these factors that confound actual changes over time due to experience.

As secondary validity checks, we revisited the demographics collected in the 37 DG studies and recovered four variables that were surveyed in many of them: age, sex, self-reported overall experience (i.e. the number of overall previous MTurk studies completed) and credulity (i.e. self-reported believing that their recipient was real ${ }^{2}$ ). We selected the first three variables because of their expected relationship with our primary variables: experience (playing DG studies) and DG giving. Specifically, we anticipate age to have a positive effect on giving (see, e.g., List, 2004) and to be positively correlated with experience; sex to also have a positive effect on giving (see, e.g., Engel, 2011) but to be uncorrelated with experience (as sex rarely changes over time); and self-reported overall experience to be positively correlated with experience and negatively correlated with giving (as per most of the articles reported in the section above). Finally, we examine the extent to which participants believe that their recipient was real to rule out the possibility that any observed experience effects are due to participants losing faith in the description of the studies. For a detailed analysis of these secondary checks, please refer to the accompanying Appendix.

\footnotetext{
${ }^{2}$ We asked the question: Unlike some other requesters on Mechanical Turk, we never use deception in our studies. Your actions and the actions of others in the study really did affect the bonuses that other individuals will earn. For our own records, to what extent did you believe that the other people were real when making your decision? Answers were on a 7-point Likert scale: "1-Very skeptical that others were real"; "7-Very confident that others were real".
} 


\section{Results}

We find clear and converging evidence of both selection and experience effects on DG giving. First, Figure 1 reveals that the greater the number of times a participant appears in our dataset, the more selfish they are in their first appearance, and that people participating in a given number of total studies get more selfish from trial to trial. It also appears that experience has less of an effect on participants who complete a greater total number of studies.

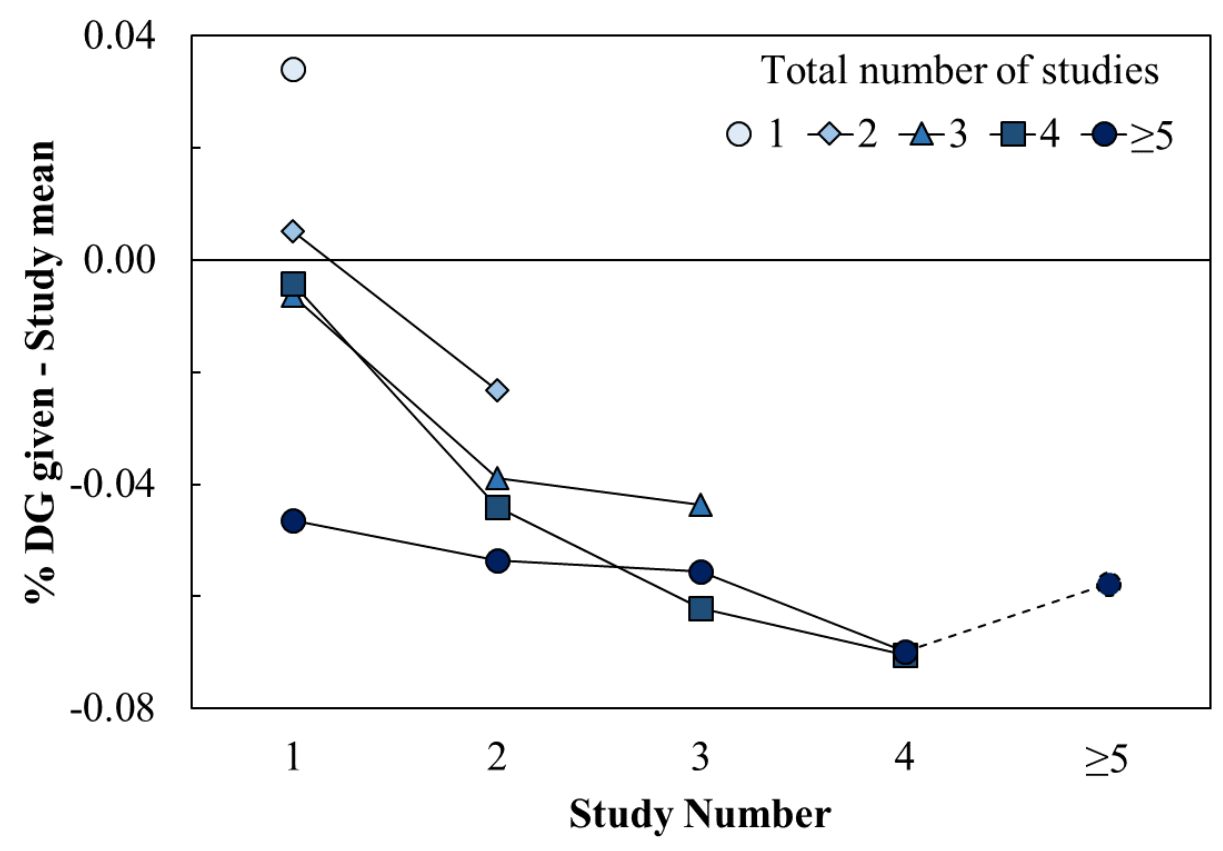

\section{Figure 1 | Fraction of DG giving as a function of study number.}

Indeed, Table 1 further confirms the decreasing effect of experience as participants complete more studies. Each model focuses on a given type of participant, based on their total number of DGs played (i.e. selection is excluded as an independent variable), and computes the effect of experience when controlling for study dummies. As can be seen, the effect tends to decrease up to the point where, for participants with 5 or more studies, the significance for experience disappears $\left(\beta_{\text {experience }}=.015, \mathrm{t}(529)=.41, \mathrm{p}=.686\right)$. We report standardized betas in text, coefficients can be found in corresponding tables. See Table $\mathrm{S} 2$ of the Online Appendix for supplementary analyses without dummies, where results for the first three models remain qualitatively similar, and where the coefficient for experience in the last model is also negative and significant. 


\begin{tabular}{lcccc}
\hline & \multicolumn{4}{c}{ Total number of games played } \\
\cline { 2 - 5 } & 2 & 3 & 4 & $\geq 5$ \\
\hline Experience & $-.031^{* * *}$ & $-.022^{* *}$ & $-.027^{* * *}$ & .002 \\
& $(.006)$ & $(.007)$ & $.007)$ & $(.005)$ \\
Constant & $.308^{* * *}$ & $.288^{* * *}$ & $.425 * * *$ & $.342^{* * *}$ \\
& $(.041)$ & $(.073)$ & $(.104)$ & $(.067)$ \\
\hline Study dummies & yes & yes & yes & yes \\
\hline $\mathrm{N}$ & 2,885 & 1,128 & 548 & 529 \\
$\mathrm{R}^{2}$ & .039 & .041 & .074 & .051 \\
\hline
\end{tabular}

Table 1 | The effect of experience on DG giving, by total number of games played. Shown are raw coefficients (b) and robust standard errors, clustered on participants' ID, in parentheses. N size corresponds to the number of clusters or participants. *** $\mathrm{p}<0.001 ; * * \mathrm{p}<0.01 ; * \mathrm{p}<0.05$.

An alternative approach that pools all participant types and has experience, selection and their interaction as independent variables ratifies our results: in the first model of Table 2 we find a negative effect of selection and experience $\left(\beta_{\text {selection }}=-.102, \mathrm{t}(17,791)=-9.12, \mathrm{p}<.001 ; \beta_{\text {experience }}=-.178, \mathrm{t}(17,791)=\right.$ -8.92, $\mathrm{p}<.001)$, and a significant and positive interaction $\left(\beta_{\text {interaction }}=.152, \mathrm{t}(17,791)=6.50, \mathrm{p}<.001\right)$, such that experience has less of an effect on participants who complete a greater total number of studies.

The model specification in Table 2 also allows us to draw additional exploratory insights when incorporating the demographics described in the previous section (we exclude self-reported overall experience from the analyses because of its correlation with DG experience, $r=.38$, but its role is concordant as shown in the accompanying Appendix).

We find that the sign, magnitude and significance of experience, selection, and their interaction remain practically unaltered when accounting for the additional demographic variables. Model 2 simply replicates the first model in the restricted dataset where demographic values are available, whereas Model 3 introduces them to the analysis. As expected, we find a significant and positive relationship for age and sex. Interestingly, credulity has also a significant and positive relationship. ${ }^{3}$

Indeed, credulity might have explained the experience effect we observe: perhaps people are being more selfish over successive DGs because they are coming to believe that they are being deceived and there is no real recipient. On the contrary, however, we find the opposite: participants' belief that the recipient is real increases over successive DGs, $\beta_{\text {experience }}=.061, \mathrm{t}(11,434)=2.76, \mathrm{p}=.006$ (we find no significance for selection nor the interaction, $\beta_{\text {selection }}=-.007, \mathrm{t}(11,434)=-0.45, \mathrm{p}=.656 ; \beta_{\text {interaction }}=.024, \mathrm{t}(11,434)=1.06$, $\mathrm{p}=.288$ ). This increase in credulity with experience is likely explained by the fact that we never use

\footnotetext{
${ }^{3}$ See Table S3 of the Online Appendix for a similar analysis without study dummies, and Table S4 for a confirmatory analysis of the third model in Table 2 that distinguishes by participant types, as in Table 1.
} 
deception, and inform participants at the end of the study that their recipient was actually real - so it seems that we are earning a reputation for conducting honest studies. Hence, it is unlikely that the drop in generosity as participants gain experience is caused by decreased belief that the recipient is real.

\begin{tabular}{lccc}
\hline & $(1)$ & $(2)$ & $(3)$ \\
\hline Experience & $-.039^{* * *}$ & $-.041^{* * *}$ & $-.045^{* * *}$ \\
Selection & $.004)$ & $(.005)$ & $.005)$ \\
& $-.015^{* * *}$ & $-.017 * * *$ & $-.018^{* * *}$ \\
Experience x Selection & $(.002)$ & $(.002)$ & $(.002)$ \\
& $.005^{* * *}$ & $.006^{* * *}$ & $.006^{* * *}$ \\
Age & $(.001)$ & $(.001)$ & $(.001)$ \\
& & & $.003^{* * *}$ \\
Female & & $.000)$ \\
& & $.033^{* * *}$ \\
Credulity & & $.005)$ \\
& & & $.023 * * *$ \\
Constant & $.370^{* * *}$ & $.001)$ \\
& $(.014)$ & $.421 * * *$ & $.235^{* * *}$ \\
Study dummies & $y e s$ & $(.007)$ & $(.011)$ \\
\hline $\mathrm{N}$ & 17,791 & $y e s$ & $y e s$ \\
$\mathrm{R}^{2}$ & .070 & 11,421 & 11,421 \\
\hline
\end{tabular}

Table 2 | The effect of experience (number of games played) and selection (total number of games played) on DG giving. Shown are raw coefficients (b) and robust standard errors, clustered on participants' ID, in parentheses. $\mathrm{N}$ size corresponds to the number of clusters or participants. $* * *$ $\mathrm{p}<0.001 ; * * \mathrm{p}<0.01 ; * \mathrm{p}<0.05$.

\section{Discussion and Conclusions}

By means of a longitudinal dataset covering six years and 27,266 records, this brief report has documented evidence of selection and learning in DG giving. While on average selfish people tend to be more likely to participate in our DG studies, we also see that, on average, giving decreases across successive trials for a given subject - people seem to be learning to be selfish. This learning is smaller for subjects who participate in more studies (and thus are more selfish at baseline). Nonetheless, even the most experienced participants tend to give positive amounts to anonymous recipients.

What might subjects be learning, given the non-strategic nature of the DG? One possibility is that preferences are not stable and that something about exposure to DG experiments undermines prosocial preferences (e.g. reduces inequity aversion). Alternatively, it could be that non-outcome-based preferences play a part in DG giving (as suggested, for example, by Capraro \& Rand, 2018), allowing participants' beliefs to influence their giving despite the fact that their co-player takes no action. For example, if giving 
is driven by preferences for type-based reciprocity (Levine, 1998), then changes in beliefs about the recipient's type could result in a change in DG giving - and could also tie in with our findings regarding credulity. Or, if giving is driven by a preference for norm compliance (Krupka \& Weber, 2013), then changes in beliefs about what behavior is normative in the context of MTurk experiments could also affect DG giving. Another possibility is that subjects are engaging in moral licensing, and feeling less obligated to give in later studies because of what they gave in earlier studies; however, given that the median time between studies was 12 weeks and most MTurk workers complete many studies per week (e.g. 20 per week, according to Rand et al., 2014), it seems unlikely that subjects were connecting behavior in one DG to their choices in a past DG (for example, the size of endowment in the previous game does not affect giving in the current game, Table S5 of the Online Appendix).

Why do subjects who participate in more studies give less on average, even in the first game? One possibility is that altruistic people might feel bad or conflicted about taking such decisions and opt to avoid further DG games. Another potential explanation is that those who are more active on MTurk (and thus participate in more studies) are more inclined to approach the games with an interest in maximizing their payoffs and earning more money. As with laboratory experiments, any experience subjects have prior to the first game is unknown. The studies here gathered were exhaustive for our requester account, but certainly participants could have, and likely did, participate in similar games elsewhere. Indeed, subjects who participated in more games in our dataset also self-reported more prior experience when they first entered our dataset $(r=.19, \mathrm{p}<.001 ; \log -10$ transformed due to right skew), and consistent with our results reported above, an exploratory analysis predicting DG giving in the first game using both self-reported experience and total number of games played finds that both are negative related to giving $\left(\beta_{\text {totalDG }}=-.042\right.$, $\left.\mathrm{t}(11,027)=-4.39, \mathrm{p}<.001 ; \beta_{\text {self-reported }}=-.146, \mathrm{t}(11,027)=-14.18, \mathrm{p}<.001\right)$.

We believe that this brief report has increased our understanding of the relationship between experience, selection. and giving in a non-strategic setting. For this, we have shown a negative effect of experience using a large and diverse longitudinal sample of participants, in a time horizon rarely captured before. We hope that the findings encourage further research that explores the extent to which learning and selection are driven by changes in preferences per se versus the combination of changing beliefs and nonoutcome-based preferences. 


\section{References}

Achtziger, A., Alós-Ferrer, C., \& Wagner, A. K. (2015). Money, depletion, and prosociality in the dictator game. Journal of Neuroscience, Psychology, and Economics, 8(1), 1.

Arechar, A.A., Kraft-Todd, G., Rand, D.G. (2017). Turking Overtime: How Participant Characteristics and Behavior Vary Over Time and Day on Amazon Mechanical Turk. Journal of the Economic Science Association. 3(1). 1-11.

Benndorf, V., Moellers, C., Normann, H.T. (2017). Experienced vs. inexperienced participants in the lab: do they behave differently? Journal of the Economic Science Association. 3(1). 12-25.

Brañas-Garza, P., Kovárík, J., \& Neyse, L. (2013). Second-to-fourth digit ratio has a non-monotonic impact on altruism. PloS One, 8(4), e60419.

Brosig-Koch, J., Riechmann, T., \& Weimann, J. (2017). The dynamics of behavior in modified dictator games. PloS One, 12(4), e0176199.

Camerer, C. F., \& Thaler, R. H. (1995). Anomalies: Ultimatums, dictators and manners. Journal of Economic Perspectives, 9(2), 209-219.

Capraro, V., Jordan, J.J., Rand, D.G. (2014). Heuristics guide the implementation of social preferences in one-shot Prisoner's Dilemma experiments. Scientific Reports. 4(6790).

Capraro, V., Rand, D.G. (2018). Do the Right Thing: Experimental evidence that preferences for moral behavior, rather than equity or efficiency per se, drive human. Judgment and Decision Making. 13(1). 99111.

Conte, A., Levati, M.V., Montinari, N. (2019). Experience in public goods experiments. Theory and Decision. 86(1). 65-93.

Engel. C. (2011). Dictator games: a meta study. Experimental Economics. 14(4). 586-610.

Fudenberg, D., Levine, D.K. (1998). The Theory of Learning in Games. Cambridge, MA: MIT Press.

Guala, F., \& Mittone, L. (2010). Paradigmatic experiments: the dictator game. The Journal of SocioEconomics, 39(5), 578-584.

Krupka, E.L. Weber, R.A. (2013). Identifying Social Norms Using Coordination Games: Why Does Dictator Game Sharing Vary? Journal of the European Economic Association, 11(3), 495-524.

Levine, D. (1998). Modeling Altruism and Spitefulness in Experiments. Review of Economic Dynamics. 1, 593-622.

List, J. A. (2004). Young, selfish and male: Field evidence of social preferences. Economic Journal, 114(492), 121-149.

Matthey, A., Regner, T. (2013). On the independence of history: experience spill-overs between experiments. Theory and Decision. 75(3), 403-419.

McAuliffe, W. H., Forster, D. E., Pedersen, E. J., \& McCullough, M. E. (2018). Experience with anonymous interactions reduces intuitive cooperation. Nature Human Behaviour, 2(12), 909-914. Also finds decay within weeks from study. In two waves.

Rand, D. G., Peysakhovich, A., Kraft-Todd, G. T., Newman, G. E., Wurzbacher, O., Nowak, M. A., \& Greene, J. D. (2014). Social heuristics shape intuitive cooperation. Nature communications, 5(1), 1-12. Sass, M., Timme, F., \& Weimann, J. (2018). On the Dynamics of Altruistic Behavior. Working Paper. 


\section{Appendix}

As detailed in the Methods Section, this Appendix assesses how the results found in our dataset align with the ones anticipated. As expected, we find in Table A1 that younger participants share relatively less than their older counterparts $(\mathrm{p}<.001)^{4}$, that females give relatively more than men $(\mathrm{p}<.001)$, that those with more self-reported experience give less than those with less experience $(\mathrm{p}<.001$; log-10 transformed due to right skew), and that those who believe more that their recipient is real give more than those who believe less $(\mathrm{p}<.001)$. We also find a negative relationship between the size of the endowment and giving, but the effect disappears once study dummies are introduced (as endowment was constant within a study).

\begin{tabular}{lccc}
\hline & Mean & Std. Deviation & $\mathrm{N}$ \\
\hline Overall & .305 & .268 & 27,266 \\
Total number of studies & & & \\
$\quad 1$ & .354 & .266 & 12,701 \\
2 & .289 & .265 & 5,770 \\
3 & .257 & .263 & 3,384 \\
4 & .245 & .259 & 2,192 \\
$\geq 5$ & .231 & .256 & 3,219 \\
Studies completed (self-reported) & & & \\
$\quad$ Below or at the median (500) & .344 & .276 & 9,684 \\
$\quad$ Above the median & .255 & .267 & \\
Sex & & & 12,010 \\
$\quad$ Female & .331 & .257 & 12.271 \\
$\quad$ Male & .273 & .277 & 11,611 \\
Age group & & & 11,593 \\
$\quad$ Below or at the median (31) & .289 & .270 & \\
$\quad$ Above the median & .320 & .267 & 14,165 \\
Size of the stake & & & 13,101 \\
$\quad$ Below or at the median $(\$ 0.30)$ & .335 & .260 & 10,254 \\
$\quad$ Above the median & .272 & .273 & 5,791 \\
Belief that the recipient is real & & & .273 \\
$\quad$ Below or at the median $(67 \%)$ & .285 & .328 &
\end{tabular}

Table A1 | Fraction of DG giving as a function of statistics of the variables used in the study.

With regards to our last validity check, we also confirm the expected patterns for age, sex, and self-reported experience. Specifically, a replication of the first model of Table 2, with the relevant variables instead of giving, reveals that experience has a significant positive relationship with age $(p<.001)$ and number of previous MTurk studies completed ( $\mathrm{p}<0.001)$, and no relationship with sex $(\mathrm{p}=.211)$.

\footnotetext{
${ }^{4}$ In-text comparisons of DG giving are based on linear regression with robust standard errors clustered on participants' ID and study dummies, where the dependent variable is giving and the independent variable is the relevant variable.
} 
Supporting Information for:

\title{
Learning to be selfish? A large-scale longitudinal analysis of Dictator games played on Amazon Mechanical Turk
}

\author{
By ANTONIO A. ARECHAR AND DAVID G. RAND \\ antonio@arechar.com, drand@mit.edu
}

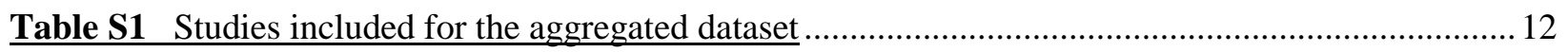

Table S2 The effect of experience on DG giving, by total number of games played ............................ 13

Table S3 The effect of experience and selection on DG giving ...................................................... 13

Table S4 The effect of experience on DG giving, by total number of games played and participants'

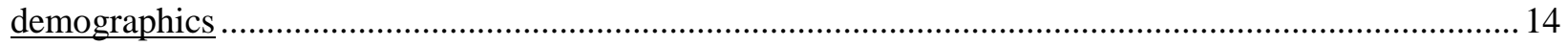

Table S5 The effect of experience, selection and endowment variation on DG giving ......................... 14 


\begin{tabular}{|c|c|c|c|c|}
\hline Study ID & $N$ & $\begin{array}{c}\text { Share of } D G \text { decisions } \\
\text { by naïve subjects }\end{array}$ & $\begin{array}{l}\text { Mean fraction given in } \\
\text { the } D G\end{array}$ & $\begin{array}{c}\text { Share of positive } \\
\text { allocations given in the } \\
D G\end{array}$ \\
\hline 01 & 436 & 0.83 & 0.36 & 0.76 \\
\hline 02 & 498 & 0.34 & 0.24 & 0.57 \\
\hline 03 & 563 & 0.70 & 0.25 & 0.76 \\
\hline 04 & 727 & 0.57 & 0.36 & 0.72 \\
\hline 05 & 1829 & 0.73 & 0.42 & 0.79 \\
\hline 06 & 1663 & 0.56 & 0.38 & 0.81 \\
\hline 07 & 2391 & 0.48 & 0.30 & 0.64 \\
\hline 08 & 1071 & 0.32 & 0.28 & 0.62 \\
\hline 09 & 440 & 0.83 & 0.39 & 0.78 \\
\hline 10 & 1258 & 0.23 & 0.23 & 0.46 \\
\hline 11 & 2213 & 0.34 & 0.25 & 0.53 \\
\hline 12 & 1795 & 0.37 & 0.23 & 0.49 \\
\hline 13 & 818 & 0.62 & 0.36 & 0.76 \\
\hline 14 & 520 & 0.50 & 0.36 & 0.71 \\
\hline 15 & 960 & 0.39 & 0.30 & 0.66 \\
\hline 16 & 563 & 0.37 & 0.16 & 0.66 \\
\hline 17 & 372 & 0.36 & 0.28 & 0.60 \\
\hline 18 & 728 & 0.47 & 0.37 & 0.79 \\
\hline 19 & 147 & 0.22 & 0.30 & 0.63 \\
\hline 20 & 524 & 0.32 & 0.23 & 0.55 \\
\hline 21 & 531 & 0.41 & 0.27 & 0.58 \\
\hline 22 & 781 & 0.55 & 0.32 & 0.67 \\
\hline 23 & 980 & 0.37 & 0.30 & 0.65 \\
\hline 24 & 335 & 0.15 & 0.24 & 0.50 \\
\hline 25 & 571 & 0.55 & 0.35 & 0.77 \\
\hline 26 & 490 & 0.61 & 0.32 & 0.69 \\
\hline 27 & 795 & 0.58 & 0.34 & 0.71 \\
\hline 28 & 31 & 0.13 & 0.23 & 0.52 \\
\hline 29 & 29 & 0.03 & 0.18 & 0.45 \\
\hline 30 & 32 & 0.16 & 0.22 & 0.41 \\
\hline 31 & 33 & 0.36 & 0.30 & 0.61 \\
\hline 32 & 275 & 0.45 & 0.33 & 0.69 \\
\hline 33 & 889 & 0.55 & 0.32 & 0.67 \\
\hline 34 & 811 & 0.34 & 0.26 & 0.56 \\
\hline 35 & 417 & 0.38 & 0.38 & 0.73 \\
\hline 36 & 552 & 0.36 & 0.29 & 0.60 \\
\hline 37 & 198 & 0.51 & 0.26 & 0.66 \\
\hline
\end{tabular}




\begin{tabular}{lcccc}
\hline & \multicolumn{4}{c}{ Total number of games played } \\
\cline { 2 - 5 } & 2 & 3 & 4 & $\geq 5$ \\
\hline Experience & $-.034^{* * *}$ & $-.028^{* *}$ & $-.031^{* * *}$ & $-.007 * *$ \\
& $(.005)$ & $(.004)$ & $(.004)$ & $(.003)$ \\
Constant & $.305^{* * *}$ & $.285^{* * *}$ & $.290^{* * *}$ & $.251^{* * *}$ \\
& $(.005)$ & $(.008)$ & $(.108)$ & $(.010)$ \\
\hline Study dummies & $n o$ & $n o$ & $n o$ & $n o$ \\
\hline $\mathrm{N}$ & 2,885 & 1,128 & 548 & 529 \\
$\mathrm{R}^{2}$ & .004 & .008 & .017 & .004 \\
\hline
\end{tabular}

Table S2 The effect of experience on DG giving, by total number of games played. Shown are raw coefficients (b) and robust standard errors, clustered on participants' ID, in parentheses. N size corresponds to the number of clusters or participants. *** $\mathrm{p}<0.001 ; * * \mathrm{p}<0.01 ; * \mathrm{p}<0.05$.

\begin{tabular}{lccc}
\hline & $(1)$ & $(2)$ & $(3)$ \\
\hline Experience & $-.049^{* * *}$ & $-.060^{* * *}$ & $-.066^{* * *}$ \\
& $(.004)$ & $.005)$ & $.005)$ \\
Selection & $-.019^{* * *}$ & $-.018^{* * *}$ & $-.018^{* * *}$ \\
& $(.002)$ & $(.002)$ & $.001)$ \\
Experience x Selection & $.007 * * *$ & $.008^{* * *}$ & $.008^{* * *}$ \\
& $(.001)$ & $(.001)$ & $.001)$ \\
Age & & & $.002^{* * *}$ \\
& & $.000)$ \\
Female & & $.041^{* * *}$ \\
& & & $.005)$ \\
Credulity & & $.019^{* * *}$ \\
& & $.001)$ \\
Constant & $.345^{* * *}$ & $.348^{* * *}$ & $.165^{* * *}$ \\
& $(.002)$ & $(.003)$ & $(.010)$ \\
\hline Study dummies & $n o$ & $n o$ & $n o$ \\
\hline $\mathrm{N}$ & 17,791 & 11,421 & 11,421 \\
$\mathrm{R}^{2}$ & .034 & .039 & .076 \\
\hline
\end{tabular}

Table S3 The effect of experience and selection on DG giving. Shown are raw coefficients (b) and robust standard errors, clustered on participants' ID, in parentheses. $N$ size corresponds to the number of clusters or participants. $* * * \mathrm{p}<0.001 ; * * \mathrm{p}<0.01 ; * \mathrm{p}<0.05$. 


\begin{tabular}{|c|c|c|c|c|c|c|c|c|}
\hline \multicolumn{5}{|c|}{ Total number of games played, with study dummies } & \multicolumn{4}{|c|}{ Total number of games played, without study dummies } \\
\hline & 2 & 3 & 4 & $\geq 5$ & 2 & 3 & 4 & $\geq 5$ \\
\hline \multirow[t]{2}{*}{ Experience } & $-.035 * * *$ & $-.024 * *$ & $-.036 * * *$ & .004 & $-.045 * * *$ & $-.039 * *$ & $-.044 * * *$ & $-.012 * * *$ \\
\hline & $(.009)$ & $(.009)$ & $(.008)$ & $(.005)$ & $(.008)$ & $(.006)$ & $(.006)$ & $(.004)$ \\
\hline \multirow[t]{2}{*}{ Age } & $.003 * * *$ & $.003 * * *$ & $.004 * * *$ & $.003 * * *$ & $.003 * * *$ & $.003 * * *$ & $.004 * * *$ & $.003 * * *$ \\
\hline & $(.000)$ & $(.001)$ & $(.001)$ & $(.001)$ & $(.000)$ & $(.001)$ & $(.001)$ & $(.001)$ \\
\hline \multirow[t]{2}{*}{ Female } & $.023 *$ & $.029 *$ & $.056^{* *}$ & .023 & $.029 * *$ & $.035^{*}$ & $.068 * * *$ & .019 \\
\hline & $(.010)$ & $(.014)$ & $(.018)$ & $(.017)$ & $(.010)$ & $(.014)$ & $(.019)$ & $(.018)$ \\
\hline \multirow[t]{2}{*}{ Credulity } & $.023 * * *$ & $.023 * * *$ & $.018^{* * *}$ & $.014 * * *$ & $.0192 * * *$ & $.019 * * *$ & $.014 * * *$ & $.010^{* *}$ \\
\hline & $(.002)$ & $(.003)$ & $(.004)$ & $(.004)$ & $(.002)$ & $(.003)$ & $(.004)$ & $(.004)$ \\
\hline \multirow[t]{2}{*}{ Constant } & $.175^{* * *}$ & $.198 * * *$ & $.194 * * *$ & $.127 * *$ & $.103^{* * *}$ & $.096^{* * *}$ & $.072 * * *$ & $.085^{* * *}$ \\
\hline & $(.026)$ & $(0.040)$ & $(.052)$ & $(.049)$ & $(.020)$ & $(.027)$ & $(.038)$ & $(.035)$ \\
\hline $\mathrm{N}$ & 2,456 & 1,075 & 538 & 526 & 2,456 & 1,075 & 538 & 526 \\
\hline $\mathrm{R}^{2}$ & .080 & .086 & .132 & .082 & .047 & .049 & .008 & .035 \\
\hline
\end{tabular}

Table S4 The effect of experience on DG giving, by total number of games played and participants' demographics. Shown are raw coefficients (b) and robust standard errors, clustered on participants' ID, in parentheses. $N$ size corresponds to the number of clusters or participants. *** $p<0.001 ; * * p<0.01 ; * p<0.05$.

\begin{tabular}{|c|c|c|}
\hline & (1) & (2) \\
\hline Experience & $\begin{array}{c}-.028 * * * \\
(.007)\end{array}$ & $\begin{array}{c}-.028 * * * \\
(.007)\end{array}$ \\
\hline Selection & $\begin{array}{c}-.007 * * \\
(.003)\end{array}$ & $\begin{array}{c}-.007 * * \\
(.003)\end{array}$ \\
\hline Experience $\mathrm{x}$ Selection & $\begin{array}{l}.003 * * \\
(.001)\end{array}$ & $\begin{array}{l}.003 * * \\
(.001)\end{array}$ \\
\hline Endowment $_{t}-$ Endowment $_{\mathrm{t}-1}$ & & $\begin{array}{l}-.0000626 \\
(.0000526)\end{array}$ \\
\hline Constant & $\begin{array}{c}.295 * * * \\
(.010)\end{array}$ & $\begin{array}{c}.296 * * * \\
(.010) \\
\end{array}$ \\
\hline Study dummies & no & no \\
\hline $\begin{array}{l}\mathrm{N} \\
\mathrm{R}^{2}\end{array}$ & $\begin{array}{c}5,090 \\
.006\end{array}$ & $\begin{array}{c}5,090 \\
006\end{array}$ \\
\hline
\end{tabular}

Table S5 The effect of experience, selection and endowment variation on DG giving. We do not include study dummies because endowment is constant across study. Shown are raw coefficients (b) and robust standard errors, clustered on participants' ID, in parentheses. N size corresponds to the number of clusters or participants. $* * * \mathrm{p}<0.001 ; * * \mathrm{p}<0.01 ; * \mathrm{p}<0.05$. 